\title{
Numerical Simulation of Liquid-Fueled Detonations by an Eulerian-Lagrangian Model
}

\author{
Hua Shen, ${ }^{1,2}$ Gang Wang, ${ }^{3}$ Kaixin Liu ${ }^{1,2, *}$ and \\ Deliang Zhang ${ }^{3}$
}

${ }^{1}$ LTCS and Department of Mechanics \& Aerospace Engineering, College of Engineering, Peking University, Beijing, China.

${ }^{2}$ Center for Applied Physics and Technology, Peking University, Beijing, China.

${ }^{3}$ LHD, Institute of Mechanics, Chinese Academy of Science, Beijing, China.

\begin{abstract}
In this paper, an Eulerian-Lagrangian twophase flow model for liquid-fueled detonations is constructed. The gaseous mixture is described by an Eulerian method, and liquid particles in gaseous mixture are traced by a Lagrangian method. An improved space-time conservation element and solution element (CE/SE) scheme is applied to the simulations of detonations in liquid $\mathrm{C}_{10} \mathrm{H}_{22}$ $\mathrm{O}_{2} /$ air systems. Different fuel droplet sizes and equivalence ratios are considered in the present study. Interestingly, the numerical results show that liquid-fueled detonations have some difference with gaseous detonations. Especially, a deficit in the propagation velocity compared to the gaseous detonation velocity is observed in mixtures with lean fuel and larger droplet sizes, while an increase in the propagation velocity compared to the gaseous detonation velocity is observed in the mixtures with very rich fuel. The surprising phenomenon is analyzed and discussed with the aid of detailed numerical results. In addition, the formation and propagation of two-phase detonation waves are characterized by series of results and the influence of particle radii is also discussed. All numerical results show that the present model can describe the gas-particle two-phase system accurately, and can be applied to numerical simulations of liquid-fueled detonations.
\end{abstract}

Keywords. Eulerian-Lagrangian model, two-phase flow, liquid-fueled detonation, CE/SE scheme.

$\operatorname{PACS}^{\circledR}($ 2010). 47.55.Ca, 47.40.Rs, 02.60.Cb.

\footnotetext{
* Corresponding author: Kaixin Liu, LTCS and Department of Mechanics \& Aerospace Engineering, College of Engineering, Peking University, 100871 Beijing, China; E-mail: kliu@pku.edu.cn.

Received: August 30, 2011. Accepted: December 07, 2011.
}

\section{Introduction}

So far, research on gaseous detonation has been increasingly improved. Although heterogeneous detonations involving particulate matter have been known in the context of mine and grain elevator explosions for a long time, systematic studies involving liquid-fueled detonations appear to have begun only in the 1950s [1]. After several decades of research, our understanding of the structure, stability, detonability, and initiation of liquid-fueled detonations is still more primitive than that of gaseous detonations. The lack of knowledge on the nature of two-phase detonations can be partly ascribed to the fact that the governing parameters of two-phase mixtures are far more than that of gaseous mixtures. Indeed, apart from the chemical composition and initial pressure and temperature of the mixture, one should take into account particle size, particle shape and particle distribution, etc. The latter effects may play a major role in liquid-fueled detonations. All these features result in mathematical difficulties arising even in the linear analysis of detonations in liquid fuel-oxidizer mixtures. Moreover, depending on the average particle size, the detonation zone thickness in liquid fuel-oxidizer mixtures is at least a few times larger than that in gaseous fuel-oxidizer mixtures (even for nearly the same composition), hence, the size of shock tubes used in liquid-fueled detonation studies must be essentially larger than that employed in gaseous detonation studies. This fact and sedimentation of particles increase the difficulty of experimental studies in liquid-fueled detonations. Thus, numerical simulations have become more and more important in the research of liquid-fueled detonations.

Numerical simulations of liquid-fueled two-phase detonations are far fewer than those of gas-phase or solid-fueled two-phase detonations. A. Burcat and S. Eidelman [2,3] conducted numerical simulations of a detonation wave in a cloud of heptane droplets and were able to separate out the numerical oscillations from the physical ones. Their simulations captured the secondary waves emitted by the droplet combustion and its propagation forward to reinforce the leading shock front. They considered droplet radii ranging from 50 to $500 \mu \mathrm{m}$ and found that the detonation velocity is inversely proportional to the width of the reaction zone behind the shock front. The work of S. A. Zhdan and D. V. Voronin $[4,5]$ has focused on simulating the initiation of liquid-fueled detonations. They have considered initiation both using a condensed explosive and using gaseous detonation in a stoichiometric hydrogen-oxygen mixture. 
Detonation initiation in monodispersed and polydispersed sprays of liquid fuels in air and oxygen with a range of droplet sizes from 50 to $700 \mu \mathrm{m}$ were considered in the study of A. A. Vasil'ev [6]. The results of these simulations have been used to develop simple models and analytical formulas for estimating the initiation energies for planar, cylindrical, and spherical detonations. In another study, S. A. Zhdan and E. S. Prokhorov [7] reported the numerical cellular structure of a cryogenic hydrogen and oxygen mixture. More recently, investigations of detonation onset in polydispersed fuel-air mixtures were carried out by N. N. Smirnov [8]. G. D. Roy [9] and K. Kailasanath [10] have made comprehensive overviews on the liquid-fueled two-phase detonations.

The numerical simulation of liquid-fueled detonation is very difficult due to the complex physical and chemical phenomena. It demands appropriate physical and chemical models and high accurate numerical methods. In present work, an Eulerian-Lagrangian two-phase flow model for liquid-fueled detonations is constructed and an improved high accurate CE/SE (space-time conservation element and solution element) scheme is adopted for the liquid-fueled detonation simulations. Detonations in liquid $\mathrm{C}_{10} \mathrm{H}_{22}-$ $\mathrm{O}_{2} /$ air systems with different fuel droplet sizes and equivalence ratios are simulated. All numerical results were compared and discussed carefully.

\section{Numerical Models}

\subsection{Two-phase Flow Model and Governing Equations}

Liquid-fueled detonation is a heterogeneous two-phase fluid dynamic process combined with chemical reactions. It contains a series of complex physical and chemical process including atomization, droplet breakup, vaporization, fuel combustion etc. The two-fluid (Eulerian-Eulerian) model is usually used in two-phase detonation simulation. The particle phase is considered as continuous medium, so continuous medium mechanics is used to describe the flow of particle phase. This makes the problem to some extent by homogenization. In the present study, an Eulerian-Lagrangian model is introduced for treating two-phase flows. In the present model, all particles are traced by a Lagrangian method. Following assumptions were made for the present model: the gas phase behaves as an ideal gas; the temperature of all gaseous species is the same; there are no process of collision, coalescence and fragmentation in particle phase; the shape of particles always keeps to be spherical even in process of separation, evaporation, etc; the temperature distribution in particles is uniform; the volume occupied by the droplets is negligible when compared with the volume of the gas; chemical reactions occur only in the gas phase; if chemical reaction occurs, the chemical energy is absorbed only by gas, which is considered as ideal.
Under above assumptions, the gas-phase is governed by Euler equations:

$$
\frac{\partial \mathbf{Q}}{\partial \mathbf{t}}+\frac{\partial \mathbf{E}}{\partial \mathbf{x}}+\frac{\partial \mathbf{F}}{\partial \mathbf{y}}=\mathbf{S} .
$$

In Equation (1), $\mathbf{Q}$ is the vector of conserved variables, $\mathbf{E}$ and $\mathbf{F}$ are conservation flux vectors in the $x$ - and $y$ directions, $\mathbf{S}$ is the source term vector.

Combined with chemical reaction and phase transition, the expressions of $\mathbf{Q}, \mathbf{E}, \mathbf{F}, \mathbf{S}$ are as follows

$\mathbf{Q}=\left(\begin{array}{c}\rho_{i} \\ \rho u \\ \rho v \\ e\end{array}\right), \mathbf{E}=\left(\begin{array}{c}\rho_{i} u \\ \rho u^{2}+p \\ \rho u v \\ (e+p) u\end{array}\right), \mathbf{F}=\left(\begin{array}{c}\rho_{i} v \\ \rho u v \\ \rho v^{2}+p \\ (e+p) v\end{array}\right)$

and

$$
\mathbf{S}=\left(\begin{array}{c}
\omega_{i}+\delta \sum_{1}^{N_{p}} \frac{I_{p k}}{d V_{k}} \\
\sum_{1}^{N_{p}-f_{x k}+u_{p k} I_{p k}} V_{k} \\
\sum_{1}^{N_{p}-f_{y k}+v_{p k} I_{p k}} \\
d V_{k}
\end{array}\right.
$$

The Lagrangian governing equations for the $k$ th particle are as follows

$$
\left\{\begin{array}{l}
\frac{d m_{p k}}{d t}=-I_{p k} \\
\frac{d x_{p k}}{d t}=u_{p k} \\
\frac{d y_{p k}}{d t}=v_{p k} \\
m_{p k} \frac{d u_{p k}}{d t}=f_{x k} \\
m_{p k} \frac{d v_{p k}}{d t}=f_{y k} \\
C_{v} \frac{d T_{p k}}{d t}=q_{d k} .
\end{array}\right.
$$

Here $\rho_{i}\left(i\right.$ from 1 to $N_{s}$ ) is the mass density of the $i$ th species (especially, $\rho_{1}$ is defined as the density of fuel gas), $N_{s}$ is the number of considered species, $\omega_{i}$ is the production rate of the $i$ th species. $\rho, u, v, p$ and $e$ are the total density, the velocity components of $x$-direction and $y$-direction, the pressure, and the total energy per unit volume of the gas phase, respectively. Accordingly, $m_{p k}, x_{p k}, y_{p k}, u_{p k}, v_{p k}$, $T_{p k}, e_{p k}$ and $I_{p k}$ denote the mass, the position coordinates components and the velocity components, the temperature, the internal energy per unit mass and atomization rate of the $k$ th ( $k$ from 1 to $N_{p}$ ) particle, respectively. $N_{p}$ is the number of initial particles which is determined by the equivalence ratio of the liquid fuel-gas mixture. $f_{x k}$ and $f_{y k}$ are the force components acting on the $k$ th particle. $q_{d k}$ is the convection heat transfer between gas mixtures and the $k$ th particle. $C_{v}$ is the specific heat capacity of the liquid fuel. $d V_{k}$ is the volume of gas phase influenced by the $k$ th particle which is related to the Euler grid. When $i=1, \delta=1$, otherwise, $\delta=0$. 
The dynamic interaction between liquid droplets and gas could result in instability in the droplet interface and atomization of droplets. According to boundary layer theory, A. A. Ranger and J. A. Nicholls [11] derived the atomization rate of the $k$ th droplet

$$
I_{p k}=2 \sqrt{3 \pi^{3}} \rho_{f}\left(\frac{\rho \mu}{\rho_{f} \mu_{f}}\right)^{\frac{1}{6}}\left(\frac{\mu}{\rho_{f}}\right)^{\frac{1}{2}} r_{k}^{\frac{3}{2}}\left|V-V_{p k}\right|^{\frac{1}{2}} .
$$

The force components acting on the $k$ th particle are expressed as $[11,12]$

$$
\left\{\begin{array}{c}
f_{x k}=0.5 \pi r_{k}^{2} C_{D k} \rho\left|V-V_{p k}\right|\left(u-u_{p k}\right) \\
f_{y k}=0.5 \pi r_{k}^{2} C_{D k} \rho\left|V-V_{p k}\right|\left(v-v_{p k}\right),
\end{array}\right.
$$

with

$$
\begin{aligned}
\left|V-V_{p k}\right| & =\left[\left(u-u_{p k}\right)^{2}+\left(v-v_{p k}\right)^{2}\right]^{1 / 2}, \\
C_{D k} & = \begin{cases}27 \operatorname{Re}_{k}^{-0.84}, & \operatorname{Re}_{k}<80 \\
0.27 \operatorname{Re}_{k}^{0.21}, & 80 \leq \operatorname{Re}_{k}<10^{4} \\
2 \quad, & \operatorname{Re}_{k} \geq 10^{4},\end{cases} \\
\operatorname{Re}_{k} & =\frac{2 \rho r_{k}\left|V-V_{p k}\right|}{\mu} .
\end{aligned}
$$

The convection heat transfer between gas mixtures and the $k$ th particle is expressed as $[11,12]$

$$
q_{d k}=2 \pi \lambda \mathrm{Nu}_{k}\left(T-T_{p k}\right),
$$

with

$$
\mathrm{Nu}_{k}=2+0.6 \operatorname{Re}_{k}^{1 / 2} \operatorname{Pr}^{1 / 3} .
$$

Here, $r_{k}$ is the radius of the $k$ th particles, $\mu$ is the viscosity coefficient of gas phase, $\lambda$ is the heat conduction coefficient of gas, $\rho_{f}$ is the density of liquid fuel, $\mu_{f}$ is the viscosity coefficient of droplet phase, $C_{D k}$ is the drag coefficient, $\mathrm{Re}_{k}, \mathrm{Nu}_{k}$ and $\mathrm{Pr}$ denote Reynolds number, Nusselt number and Prandtl number, respectively.

\subsection{Chemical Reaction Model}

$\mathrm{CO}, \mathrm{CO}_{2}$ and $\mathrm{H}_{2} \mathrm{O}$ are the main products of chemical reactions in a hydrocarbon-oxygen mixture. In the present work, in order to simplify the problem and to save computing resources, the following three main global reactions involving five species are considered

$$
\begin{array}{ll}
\text { Reaction 1: } & \mathrm{C}_{n} \mathrm{H}_{m}+\left(\frac{n}{2}+\frac{m}{4}\right) \mathrm{O}_{2} \rightarrow n \mathrm{CO}+\frac{m}{2} \mathrm{H}_{2} \mathrm{O} \\
\text { Reaction 2: } & 2 \mathrm{CO}+\mathrm{O}_{2} \rightarrow 2 \mathrm{CO}_{2} \\
\text { Reaction 3: } & \mathrm{CO}_{2} \rightarrow 2 \mathrm{CO}+\mathrm{O}_{2} .
\end{array}
$$

The expression of each chemical reaction rate $\mathrm{RP}_{k}$ is available in $[13,14]$. And the production rate of the $i$ th species $\omega_{i}$ is expressed as

$$
\omega_{i}=W_{i} \sum_{k=1}^{N_{r}}\left(v_{k i}^{\prime \prime}-v_{k i}^{\prime}\right) \mathrm{RP}_{k}
$$

where $W_{i}$ is the molecular weight of the $i$ th species, $\mathrm{Nr}$ is the number of chemical reactions, $v_{k i}^{\prime}$ and $v_{k i}^{\prime \prime}$ are the stoichiometric coefficients of the $i$ th species in the $k$ th chemical reaction.

\subsection{Solution of the Thermodynamic Parameters}

For the present model, the total energy density of gas phase $E$ is defined as

$$
E=\rho h-p+\frac{\rho}{2}\left(u^{2}+v^{2}\right),
$$

where, the total density of the gas mixture $\rho$ is denoted by $\rho=\sum_{1}^{N_{s}} \rho_{i}$, and the enthalpy $h$ is calculated by summing the enthalpy of each species

$$
h=\sum_{1}^{N_{s}} Y_{i} h_{i}
$$

where, $Y_{i}$ is the mass fraction of $i$ th species in gas phase. The specific heat, enthalpy and entropy of the $i$ th species can be calculated by the thermal polynomial equations

$$
\begin{aligned}
\frac{c_{p i}}{R_{i}}= & a_{1 i} T^{-2}+a_{2 i} T^{-1}+a_{3 i}+a_{4 i} T \\
& +a_{5 i} T^{2}+a_{6 i} T^{3}+a_{7 i} T^{4}, \\
\frac{h_{i}}{R_{i} T}= & -a_{1 i} T^{-2}+a_{2 i} T^{-1} \ln T+a_{3 i}+\frac{a_{4 i}}{2} T \\
& +\frac{a_{5 i}}{3} T^{2}+\frac{a_{6 i}}{4} T^{3}+\frac{a_{7 i}}{5} T^{4}+\frac{a_{8 i}}{T}, \\
\frac{s_{i}}{R_{i}}= & -\frac{a_{1 i}}{2} T^{-2}-a_{2 i} T^{-1}+a_{3 i} \ln T+a_{4 i} T \\
& +\frac{a_{5 i}}{2} T^{2}+\frac{a_{6 i}}{3} T^{3}+\frac{a_{7 i}}{4} T^{4}+a_{9 i},
\end{aligned}
$$

where, $a_{1}-a_{9}$ are interpolation coefficients [15].

According to Dalton's law, the total pressure $p$ is the sum of the partial pressure for each species and can be calculated with the state equation for a perfect gas

$$
p=T \sum_{i=1}^{N_{S}} \rho_{i} R_{i}=\rho R T .
$$

Here, $R_{i}$ is the gas constant of the $i$ th species, and $R$ and $T$ is the gas constant and temperature of the gas mixture, respectively. 
Combining the total energy density equation Equation (16) and the state equation Equation (21), one can obtain a function of temperature

$$
F(T)=\rho \sum_{i=1}^{N_{S}} Y_{i} h_{i}-\rho \sum_{i=1}^{N_{S}} Y_{i} R_{i} T-E+\frac{\rho}{2}\left(u^{2}+v^{2}\right)=0 .
$$

$F(T)$ is the relationship between conserved variables and thermodynamic parameters. In present study, a Newton iterative procedure is adopted to calculate the temperature

$$
\begin{aligned}
T_{n+1} & =T_{n}-\frac{F\left(T_{n}\right)}{F^{\prime}\left(T_{n}\right)}, \\
F^{\prime}\left(T_{n}\right) & =\frac{d(F(T))}{d T}=\rho \sum_{i=1}^{\mathrm{N} \_\mathrm{s}}\left(Y_{i} c_{p i}-Y_{i} R_{i}\right) \\
& =\rho\left(c_{p}-R\right) .
\end{aligned}
$$

The internal energy per unit mass of the $k$ th particle is defined as

$$
e_{p k}=C_{v} T_{p k}
$$

\section{The Improved CE/SE Scheme}

The CE/SE method was originally proposed by S. C. Chang and co-workers $[16,17]$. It is a completely new numerical framework for solving hyperbolic conservation equations. This new approach differs substantially in both concept and methodology from the well-established methods including finite difference, finite volume, finite element, and spectral methods etc. It has many nontraditional features and advantages, including unified treatment of space and time, satisfying both local and global flux conservations in space and time, high accuracy for solving hyperbolic conservation equations, and the simple mathematical treatment. Moreover, Riemann solvers are not needed to capture shocks, and dimensional splitting method is not needed in the multidimensional schemes.

The CE/SE scheme depends on the definitions of conservation elements (CE) and solution elements (SE). Different definitions of $\mathrm{CE}$ and $\mathrm{SE}$ lead to different numerical schemes. In Chang's original scheme, the number of CEs is consistent with the number of unknowns designated by the scheme including the mesh variables and their spatial derivatives. Therefore, two CEs are needed at each grid point to derive two discrete equations for $Q$ and $Q_{x}$ in a one-dimensional case. Similarly, three and four CEs are needed at each grid point in two- and three-dimensional cases, respectively.

In the present paper, a simple type of $\mathrm{CE}$ and $\mathrm{SE}$ on the general hexahedrons mesh was adopted. The

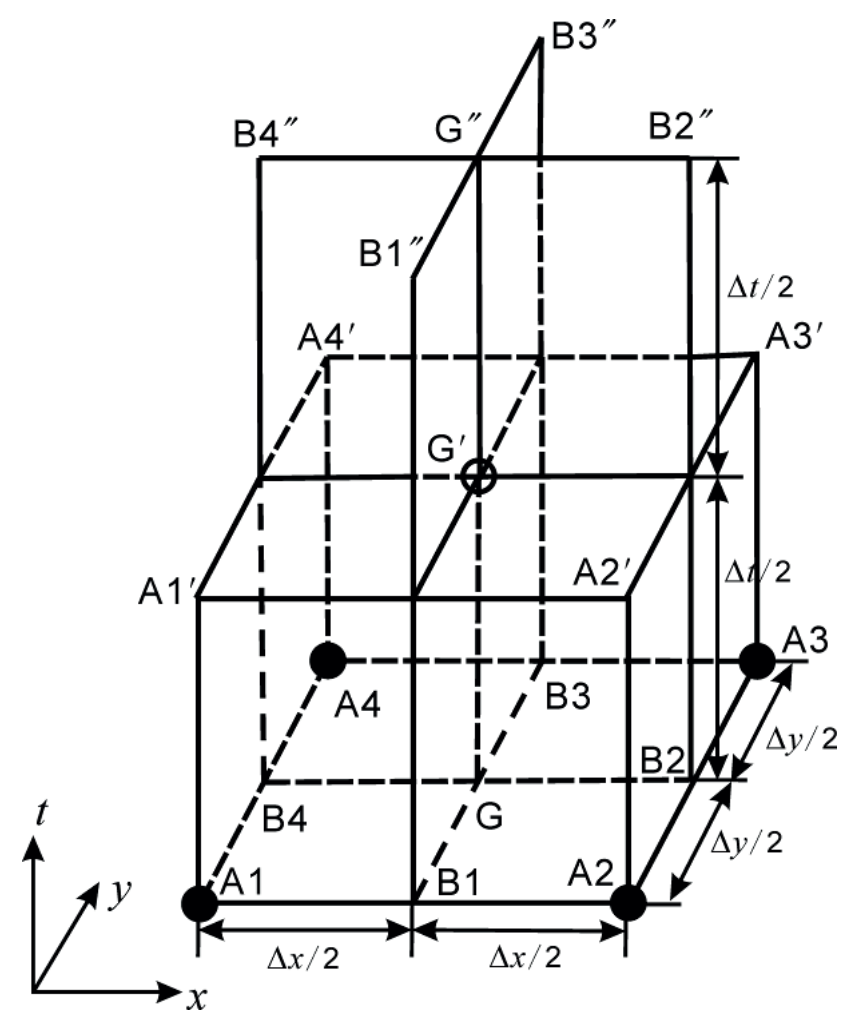

Figure 1. Space-time geometrical configuration of the improved two-dimensional CE/SE scheme.

conservation element $\mathrm{CE}\left(G^{\prime}\right)$ is defined by hexahedron $A 1 A 2 A 3 A 4 A 1^{\prime} A 2^{\prime} A 3^{\prime} A 4^{\prime}$, the solution element $\operatorname{SE}\left(G^{\prime}\right)$ constituted by the three vertical planes $\left(B 1 B 3 B 3^{\prime \prime} B 1^{\prime \prime}\right.$, $B 2 B 4 B 4^{\prime \prime} B 2^{\prime \prime}$ and $\left.A 1^{\prime} A 2^{\prime} A 3^{\prime} A 4^{\prime}\right)$ intersecting at $G^{\prime}$ and their neighborhood space as demonstrated in Figure 1. Based on the present definition of SE and CE, an improved $\mathrm{CE} / \mathrm{SE}$ scheme can be easy to construct and extend to threedimensional situation directly.

Let $x_{1}=x, x_{2}=y$ and $x_{3}=t$ be considered as the coordinates of a three-dimensional Euclidean space $E_{3}$. Because Equation (1) can be expressed as $\nabla \cdot \vec{h}=S$ with $\vec{h}=(E, F, Q)$. Gauss' divergence theorem in the spacetime $E_{3}$ implies that Equation (1) is the differential form of the integral conservation law

$$
\oiint_{S(V)} \vec{h} \cdot \vec{d} s=0
$$

where $S(V)$ is the boundary of an arbitrary space-time region $V$ in $E_{3}$, and $\vec{d} s=d \sigma \vec{n}$ with $d \sigma$ and $\vec{n}$, respectively, being the area and the unit outward normal vector of a boundary element on $S(V)$.

Assume that the integral conservation laws are satisfied in the conservation element $\mathrm{CE}\left(G^{\prime}\right)$ which is defined as demonstrated in Figure 1. Note that the outward unit normal vectors of $A 1^{\prime} A 2^{\prime} A 3^{\prime} A 4^{\prime}, A 1 A 2 A 3 A 4, A 2 A 3 A 3^{\prime} A 2^{\prime}$, 
$A 1 A 4 A 4^{\prime} A 1^{\prime}, A 1 A 2 A 2^{\prime} A 1^{\prime}$ and $A 3 A 4 A 4^{\prime} A 3^{\prime}$ are $(0,0,1)$, $(0,0,-1),(1,0,0),(-1,0,0),(0,-1,0)$ and $(0,1,0)$, respectively. Thus Equation (26) is equivalent to

$$
\begin{aligned}
& \oiint_{S(V)} \vec{d} s \\
& =\iint_{A 1^{\prime} A 2^{\prime} A 3^{\prime} A 4^{\prime}} Q d x d y-\iint_{A 1 A 2 A 3 A 4} Q d x d y \\
& \quad+\iint_{A 2 A 3 A 3^{\prime} A 2^{\prime}} E d y d t-\iint_{A 1 A 4 A 4^{\prime} A 1^{\prime}} E d y d t \\
& \quad-\iint_{A 1 A 2 A 2^{\prime} A 1^{\prime}} F d x d t-\iint_{A 3 A 4 A 4^{\prime} A 3^{\prime}} F d x d t=0
\end{aligned}
$$

The physical variables in $\operatorname{SE}\left(G^{\prime}\right)$ are approximated by the first-order Taylor expansions at $G^{\prime}$. Note that, $\operatorname{CE}\left(G^{\prime}\right)$ is related to not only $\operatorname{SE}\left(G^{\prime}\right)$ but also the $\operatorname{SEs}$ of $\operatorname{SE}(A 1)$, $\mathrm{SE}(A 2), \operatorname{SE}(A 3)$ and $\mathrm{SE}(A 4)$. With the aid of first-order Taylor expansions in the five SEs related to $\operatorname{CE}\left(G^{\prime}\right)$, Equation (27) is equivalent to

$$
Q_{G^{\prime}}-\frac{\Delta t}{4} S_{G^{\prime}}=\frac{1}{4}\left(\bar{Q}+\frac{\Delta t}{\Delta x} \bar{E}+\frac{\Delta t}{\Delta y} \bar{F}+\frac{\Delta t}{4} \bar{S}\right)
$$

where

$$
\left\{\begin{aligned}
\bar{Q}= & Q\left(\frac{\Delta x}{4}, \frac{\Delta y}{4}, 0\right)_{A 1}+Q\left(-\frac{\Delta x}{4}, \frac{\Delta y}{4}, 0\right)_{A 2} \\
& +Q\left(-\frac{\Delta x}{4},-\frac{\Delta y}{4}, 0\right)_{A 3}+Q\left(\frac{\Delta x}{4},-\frac{\Delta y}{4}, 0\right)_{A 4} \\
\bar{E}= & E\left(0, \frac{\Delta y}{4}, \frac{\Delta t}{4}\right)_{A 1}-E\left(0, \frac{\Delta y}{4}, \frac{\Delta t}{4}\right)_{A 2} \\
& -E\left(0,-\frac{\Delta y}{4}, \frac{\Delta t}{4}\right)_{A 3}+E\left(0,-\frac{\Delta y}{4}, \frac{\Delta t}{4}\right)_{A 4} \\
\bar{F}= & F\left(\frac{\Delta x}{4}, 0, \frac{\Delta t}{4}\right)_{A 1}+F\left(-\frac{\Delta x}{4}, 0, \frac{\Delta t}{4}\right)_{A 2} \\
& -F\left(-\frac{\Delta x}{4}, 0, \frac{\Delta t}{4}\right)_{A 3}-F\left(\frac{\Delta x}{4}, 0, \frac{\Delta t}{4}\right)_{A 4} \\
\bar{S}= & S_{A 1}+S_{A 2}+S_{A 3}+S_{A 4} .
\end{aligned}\right.
$$

Here, $X(d x, d y, d t)_{N}$ denotes the Taylor expansions of $Q$, $E$ and $F$ at the reference point $N$, i.e.

$$
\begin{aligned}
X(d x, d y, d t)_{N}= & X_{N}+\left(X_{x}\right)_{N} d x \\
& +\left(X_{y}\right)_{N} d y+\left(X_{t}\right)_{N} d t
\end{aligned}
$$

Substituting Equation (30) into Equation (1), we obtain

$$
\left(Q_{t}\right)_{N}=-\left(E_{x}\right)_{N}-\left(F_{y}\right)_{N} .
$$

Equations (30) and (31) imply that the variables needed to be calculated are $Q, Q_{x}$ and $Q_{y}$ at each grid point, because $E$ and $F$ are functions of $Q$.
Using the continuous conditions at point $A 1^{\prime}, A 2^{\prime}, A 3^{\prime}$ and $A 4^{\prime}$, we have the derivatives of $Q$ with respect to $x$ and $y$

$$
\begin{aligned}
\left(Q_{x}\right)_{G^{\prime}} & =W\left[\left(Q_{x}\right)_{G^{\prime}}^{-},\left(Q_{x}\right)_{G^{\prime}}^{+}, \alpha\right],\left(Q_{y}\right)_{G^{\prime}} \\
& =W\left[\left(Q_{y}\right)_{G^{\prime}}^{-},\left(Q_{y}\right)_{G^{\prime}}^{+}, \alpha\right]
\end{aligned}
$$

where

$$
\left\{\begin{aligned}
\left(Q_{x}\right)_{G^{\prime}}^{-}= & -\frac{1}{\Delta x}\left[Q\left(0,0, \frac{\Delta t}{2}\right)_{A 1}\right. \\
& \left.+Q\left(0,0, \frac{\Delta t}{2}\right)_{A 4}-2(Q)_{G^{\prime}}\right] \\
\left(Q_{x}\right)_{G^{\prime}}^{+}= & +\frac{1}{\Delta x}\left[Q\left(0,0, \frac{\Delta t}{2}\right)_{A 2}\right. \\
& \left.+Q\left(0,0, \frac{\Delta t}{2}\right)_{A 3}-2(Q)_{G^{\prime}}\right] \\
\left(Q_{y}\right)_{G^{\prime}}^{-}= & -\frac{1}{\Delta y}\left[Q\left(0,0, \frac{\Delta t}{2}\right)_{A 1}\right. \\
& \left.+Q\left(0,0, \frac{\Delta t}{2}\right)_{A 2}-2(Q)_{G^{\prime}}\right] \\
\left(Q_{y}\right)_{G^{\prime}}^{+}= & +\frac{1}{\Delta y}\left[Q\left(0,0, \frac{\Delta t}{2}\right)_{A 3}\right. \\
& \left.+Q\left(0,0, \frac{\Delta t}{2}\right)_{A 4}-2(Q)_{G^{\prime}}\right] .
\end{aligned}\right.
$$

The weighted average function is defined as

$$
W\left[x_{-}, x_{+}, \alpha\right]=\frac{\left|x_{+}\right|^{\alpha} x_{-}+\left|x_{-}\right|^{\alpha} x_{+}}{\left|x_{+}\right|^{\alpha}+\left|x_{-}\right|^{\alpha}}
$$

where $\alpha$ is an adjustable constant and usually equals $1 \sim 2$.

It should be noted that $Q_{G^{\prime}}$ can not be obtained explicitly from Equation (28) due to the source term $S_{G^{\prime}}$ is a function of $Q_{G^{\prime}}$. A local Newton iterative procedure is usually needed to determine $Q_{G^{\prime}}$. In the present work, to avoid the iterative procedure and save computation time, $S_{G^{\prime}}$ is replaced by its linear prediction of current time, i.e.,

$$
\left\{\begin{array}{l}
Q_{G^{\prime}}=\frac{1}{4} \bar{Q}+\frac{\Delta t}{4 \Delta x} \bar{E}+\frac{\Delta t}{4 \Delta y} \bar{F}+\frac{\Delta t}{2} \tilde{S} \\
\tilde{S}=\bar{S}+\frac{\Delta t}{2} \bar{S}_{t},
\end{array}\right.
$$

where $\bar{S}_{t}$ is the time derivative of $\bar{S}$.

More detailed description of the improved CE/SE scheme is available in Reference [18-20]. To date, the improved CE/SE method has achieved great success in the simulation of gaseous detonation [18, 20,21], two-phase detonation [20], multi-material elastic-plastic flows [19,22], spall fracture [22], etc.

\section{Numerical Results and Discussions}

In this section, planar detonations in liquid $\mathrm{C}_{10} \mathrm{H}_{22}-\mathrm{O}_{2} /$ air systems with different fuel droplet radii and equivalence ratios $(\Phi)$ are simulated. Initial pressure and temperature of the mixtures are $1 \mathrm{~atm}$ and $298 \mathrm{~K}$, respectively. Tube length is $3 \mathrm{~m}$. Detonation wave is generated by igniting in the left 


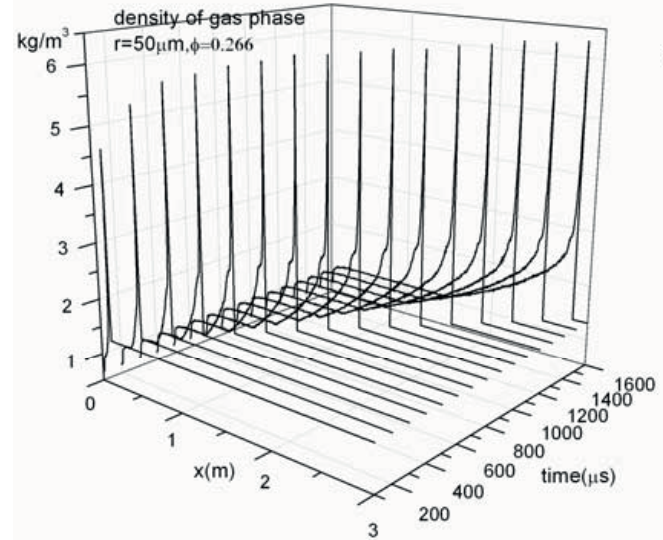

(a) the density of gas phase

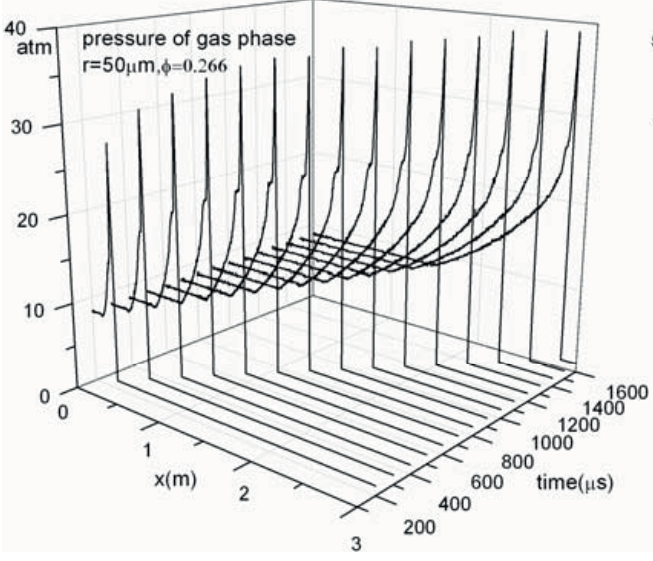

(c) the pressure of gas phase

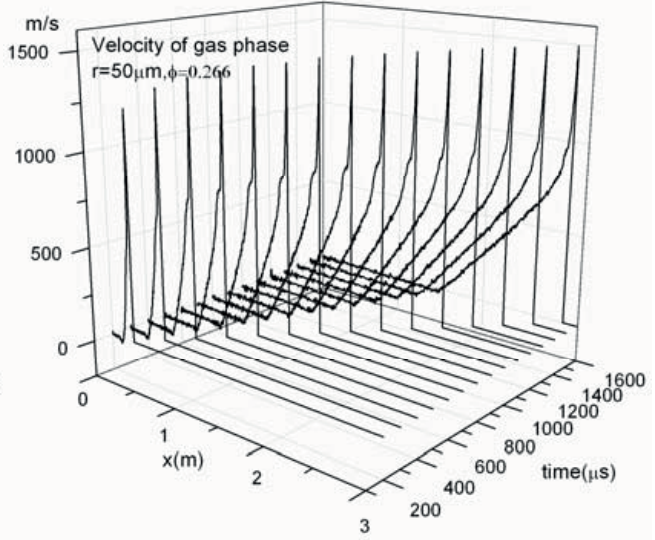

(b) the velocity of gas phase

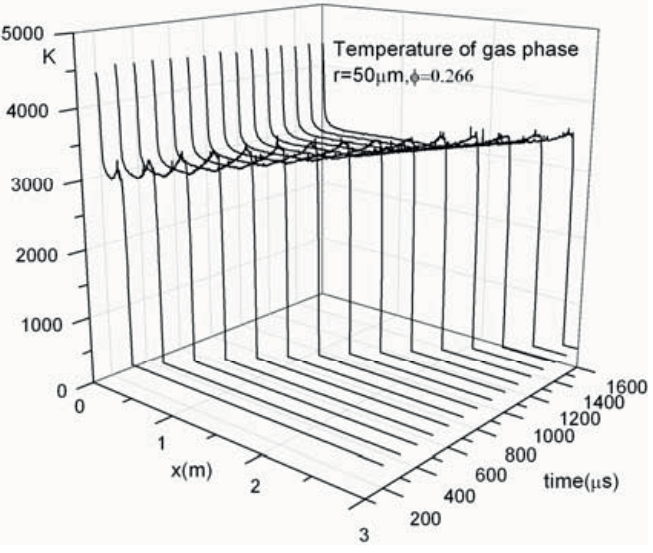

(d) the temperature of gas phase

Figure 2. Physical quantity profiles of gas phase at different times (3000 mesh grids).

with a high initial pressure and temperature as $30 \mathrm{~atm}$ and $2980 \mathrm{~K}$, respectively. The other computing parameters are given as below: $\lambda=0.1 \mathrm{~W} \cdot \mathrm{K}^{-1} \cdot \mathrm{m}^{-1}, \rho_{f}=730 \mathrm{~kg} / \mathrm{m}^{3}$, $\mu=2.07 \times 10^{-5} \mathrm{~Pa} \cdot \mathrm{s}, \mu_{f}=3.5 \times 10^{-4} \mathrm{~Pa} \cdot \mathrm{s}, C_{v}=$ $2.1 \times 10^{3} \mathrm{~J} \cdot \mathrm{kg}^{-1} \cdot \mathrm{K}^{-1}, \mathrm{Pr}=0.74$. In the present simulations, the velocity and temperature of the particles are set to 0 if the radius of the particle is decreased to 0 .

\subsection{Formation and Propagation of the Detonation Waves}

To illustrate the capability of the present model, detonations in liquid $\mathrm{C}_{10} \mathrm{H}_{22}-\mathrm{O}_{2}$ system is simulated first by 3000 mesh grids. The initial number and radii of particles are 1917 and $50 \mu \mathrm{m}$, which is equivalent to $\Phi=0.266$. Compared with experimental study, one of the main advantages of numerical simulation is that the local and global profiles of physical variables are available at arbitrary time. To enhance the visual effect, the formation and propagation of the selfsustained detonation wave (DW) is characterized by series of results at different times. Figure 2(a)-(d) shows the distributions of the density, the velocity, the pressure and the temperature of gas phase along $x$-axis at different times. It can be seen that the DW continuously increased initially, and tends to steady state at about 300-400 $\mu$ s. For the similar ignition condition, the DW in stoichiometric $\mathrm{H}_{2}-\mathrm{O}_{2}$ mixture tends to steady state within $1 \mu \mathrm{s}$. In the present model, there are some local oscillations in the temperature curves of gas phase due to the heterogeneous influence of the liquid particles. This is different from the two-fluid model [20] and more consistent with the facts. Figure 3 (a)-(c) shows the radii, the velocity, and the temperature of particles at different times. At the DW front, the velocity and temperature of the particles increase under the action of the drag force and convection heat transfer, respectively. But the radii of particles decrease due to the atomization. The peak velocity and temperature of particles significantly lower than that of gas phase. It is because the acceleration process and the heating process of particles are slower than the atomization process of particles. Figure 4 shows the mass fraction of each species in gas mixtures. The mass fraction of $\mathrm{C}_{10} \mathrm{H}_{22}$ keeps zero because the chemical reaction rate is much faster than the atomization rate of the fuel particle and the $\mathrm{O}_{2}$ is rich in this case. 


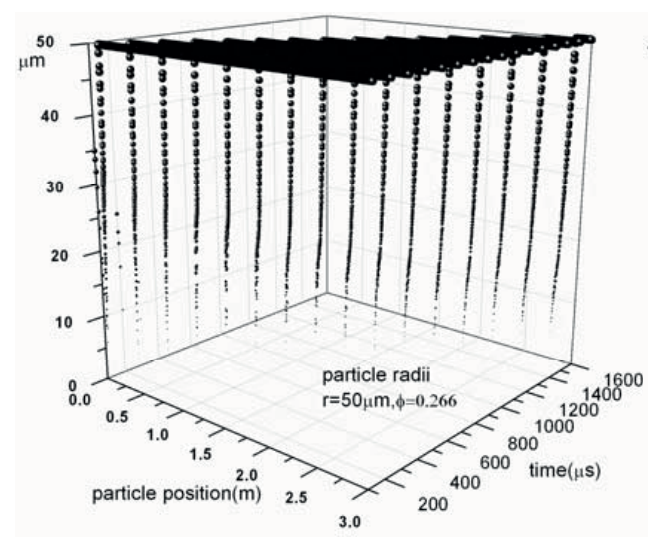

(a) the radii of particles

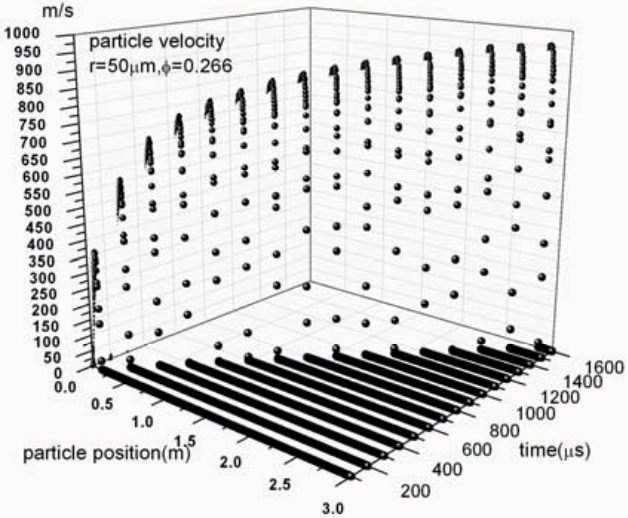

(b) the velocity of particles

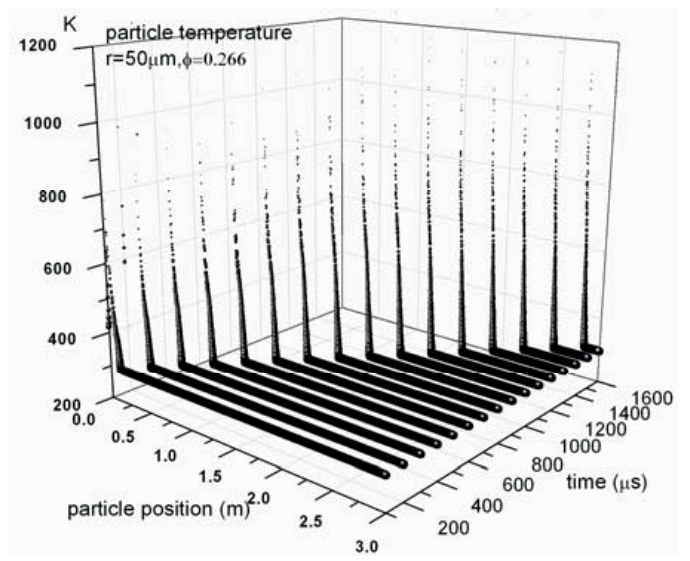

(c) the temperature of particles

Figure 3. Physical quantity profiles of particles at different times.

To check the mesh convergence of the present model, the DW is calculated by two types of finer mesh grids under the same condition. Figure 5 shows the comparison of pressure profiles of gas phase calculated by 6000 mesh grids and 9000 mesh grids. It can be seen that the results calculated by three different mesh sizes are in good agreement.

\subsection{Detonation velocity}

Theoretical, computational, and experimental studies all have shown that if the droplets are fine enough (less than about $10 \mu \mathrm{m}$ ), detonations propagating at about the equivalent gas-phase Chapman-Jouguet detonation velocity can be attained in a variety of fuel-oxygen systems. However, there is a deficit in the two-phase detonation velocity compared to the gaseous detonation velocity if droplet size is lager. Various explanations for this deficit are presented. Since the reaction zone length in two-phase detonations can be quite large, a significant velocity deficit due to wall losses is to be expected. The velocity deficit due to losses of heat and drag can be approximated using the analytical expression derived by K. W. Ragland et al. [23] for a one-dimensional two-phase detonation in a shock tube.

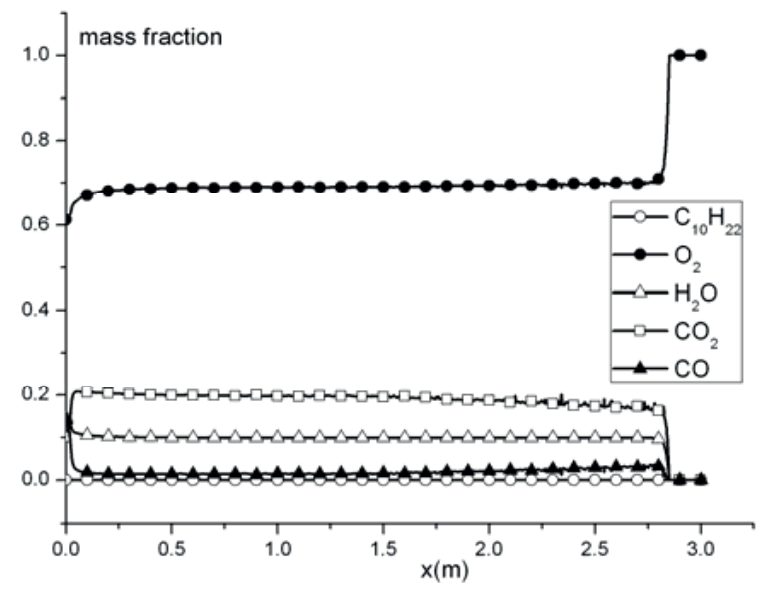

Figure 4. The mass fraction of each species in gas mixtures.

S. A. Gubin and M. Sichel [24] suggested that the deficit of the detonation velocity is not due to the heat and drag losses from the reaction zone but is mainly due to incomplete fuel reaction between the shock and $\mathrm{C}-\mathrm{J}$ plane. M. J. Tang and J. A. Nicholls et al. [25] has systematically studied the det- 


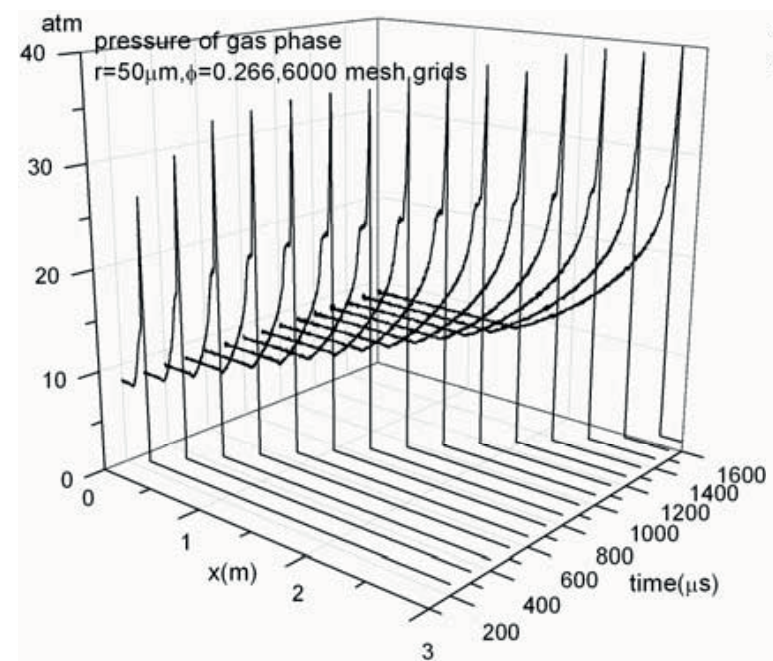

(a) the pressure calculated by 6000 mesh grids

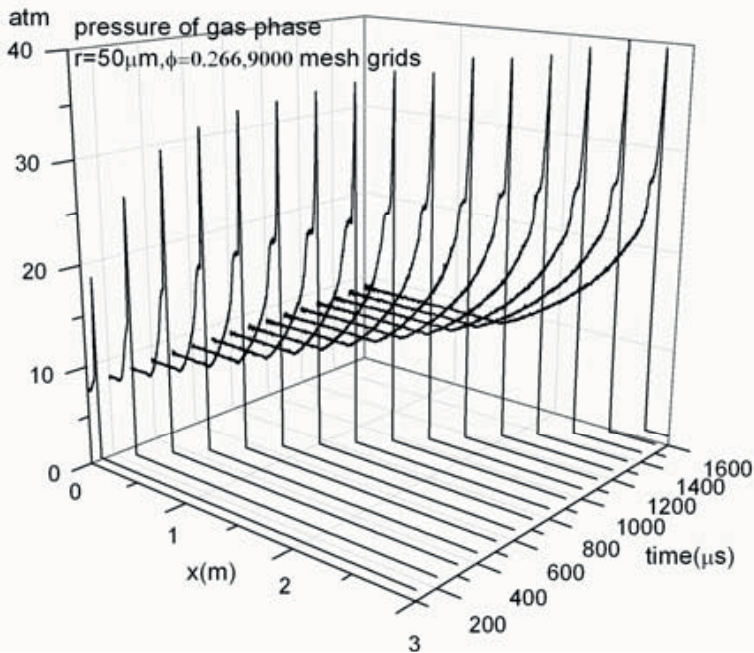

(b) the pressure calculated by 9000 mesh grids

Figure 5. Comparison of pressure profiles calculated by two different mesh sizes.

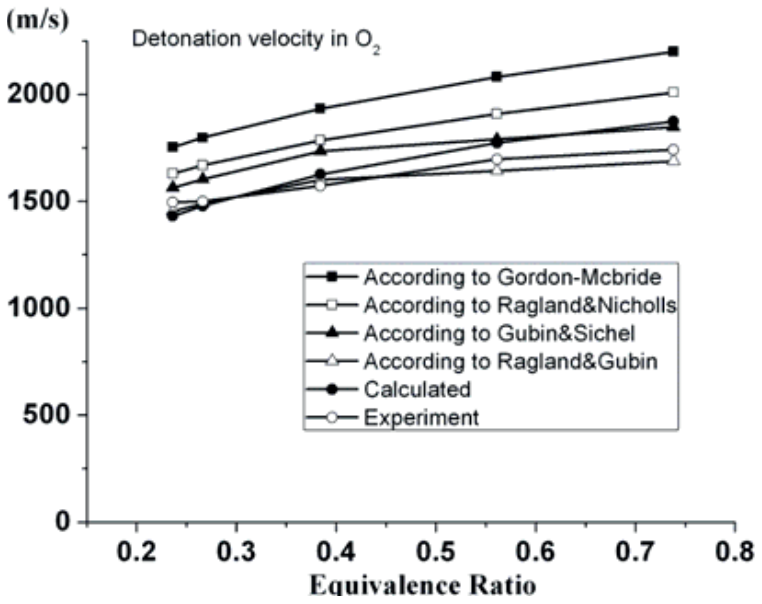

(a) detonation velocity in fuel-oxygen systems

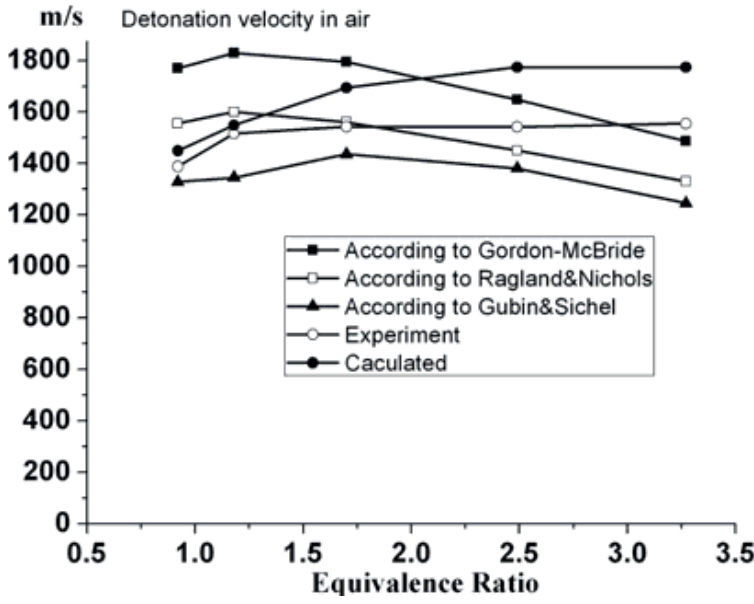

(b) detonation velocity in fuel-air systems

Figure 6. Comparison of detonation velocity in fuel-oxygen/air systems derived by different methods.

onations in a $\mathrm{C}_{10} \mathrm{H}_{22}$ spray with $200 \mu \mathrm{m}$ radius droplets in air and oxygen using a vertical shock tube. In order to verify the accuracy of the present model, the detonations in a $\mathrm{C}_{10} \mathrm{H}_{22}$ spray with $200 \mu \mathrm{m}$ radius droplets in air and oxygen are simulated. The numerical results are compared with theoretical and experimental results mentioned above. Figure 6(a) and (b) shows the detonation velocity in fueloxygen/air systems with different equivalence ratios and $200 \mu \mathrm{m}$ radius droplets derived by different methods. Theoretical values of the detonation parameters in an all-gaseous mixture in air and in oxygen were calculated using the Bordon McBride computer code [25,26]. It can be seen that the theoretical predictions are agree well with experiment results for mixtures with lean fuel. The value predicted by S. A. Gubin and M. Sichel is more accuracy than that predicted by K. W. Ragland and J. A. Nicholls. The value predicted by combining the views of K. W. Ragland and S. A. Gubin together is the most accurate. So the deficit of the detonation velocity is not only due to the heat and drag losses in the reaction zone but also the incomplete fuel reaction between the shock and $\mathrm{C}-\mathrm{J}$ plane. It is also can be found that, for rich fuel mixtures, all the theoretical values are different with experimental value in trends. The experimental data show that detonation velocity increases with equivalence ratio rapidly in lean fuel mixtures, and then approaches a constant value in rich fuel mixtures. Surprisingly, when $\Phi>3.0$, the two-phase detonation velocity in air is even higher than gaseous detonation velocity. The calculated detonation velocity using the present model is consistent with the experimental value in trends. And the 
calculated detonation velocity is a little higher than experimental detonation velocity, which may be due to the heat and drag losses in the reaction zone are not considered in the present model. Figure 7 shows the comparison of calculated detonation velocity with $\mathrm{C}-\mathrm{J}$ theory for all gaseous mixtures. It can be seen that the detonation velocity in $\mathrm{O}_{2}$ has a similar trend as that in air. Just the detonation velocity in $\mathrm{O}_{2}$ is higher than that in air which is due to the dilution effect of nitrogen in air.

Why the trend of two-phase detonation velocity in lean fuel mixtures is completely different from that in rich fuel mixtures? It is due to the difference between the global equivalence ratio and the efficient equivalence ratio of the gas-phase mixtures which sustains the detonation propagation. Figure 8 shows the particle radii distribution in the detonation propagation process. It can be seen that the particles are not completely atomized between the shock and $\mathrm{C}-\mathrm{J}$ plane. Indeed, the mass of the particle phase is reduced by only about $50 \%$ between the shock and $\mathrm{C}-\mathrm{J}$ plane. According to S. A. Gubin and M. Sichel's study, the particles atomized behind the $\mathrm{C}-\mathrm{J}$ plane almost have no contribution to the detonation velocity. For lean fuel mixtures, the fuel atomization is incomplete between the shock and $\mathrm{C}-$ $\mathrm{J}$ plane, i.e. a deficit of efficient energy release is caused, so there is a deficit of detonation velocity. In contrast, for mixtures with rich fuel, the fuel is excess for the oxygen, and the atomized fuel vapors between the shock and C-J plane are adequate for chemical reactions. Thus, there is no deficit of energy which sustains the detonation propagation. The chemical energy is absorbed only by gas mixtures, and the convection heat transfer is very slow between the gas phase and particles as mentioned in Section 4.1. Thus, the energy density of gas mixtures is greater than that of all gas mixtures with the same global equivalence ratio, and that causes the detonation velocity in two-phase mixtures with very rich fuel is even higher than that in gaseous mixtures. Figure 9 shows temperature profiles of the gas phase with different equivalence ratios. The peak temperature is behind the detonation front in lean fuel mixtures. But the peak temperature appears just at the detonation front in rich fuel mixtures. The peak temperature increases with equivalence ratio rapidly at first, and then approaches a constant value. But the temperature of final products increases with equivalence ratio at first, and then decreases. It is also because that the fuel atomization is incomplete between the shock and C-J plane. For lean fuel mixtures, the atomized fuel vapors behind the $\mathrm{C}-\mathrm{J}$ plane also participate in exothermic reactions which cause an increase of temperature. So the peak temperature is behind the detonation front and the temperature of final products increases with equivalence ratio in lean fuel mixtures. With respect to rich fuel mixtures, exothermic reactions complete between the shock and $\mathrm{C}-\mathrm{J}$ plane. The atomized fuel vapors behind the $\mathrm{C}-\mathrm{J}$ plane do not participate in any exothermic reactions but play the role of dilution. So the peak temperature appears just at the det-

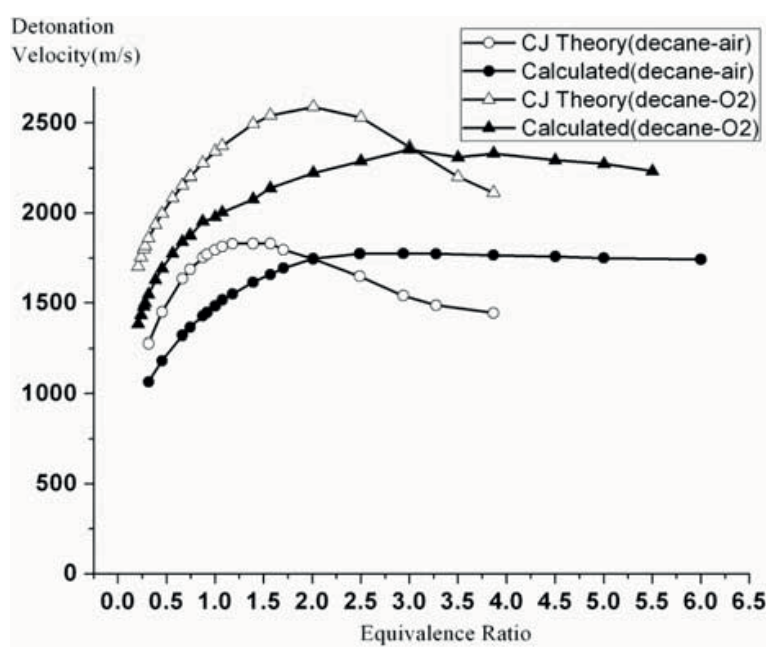

Figure 7. Comparison of calculated detonation velocity with $\mathrm{C}-\mathrm{J}$ theory for all gaseous mixtures.

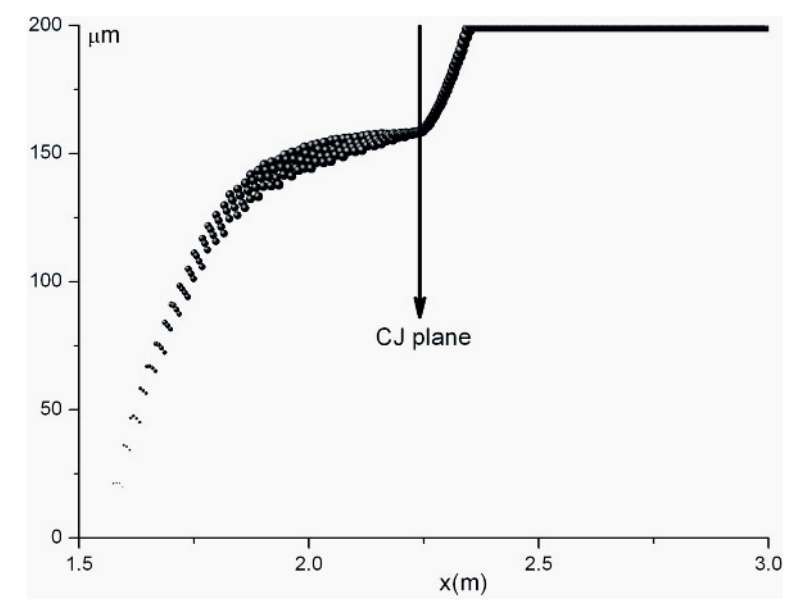

Figure 8. Particle radii distribution at $t=1900 \mu \mathrm{s}$.

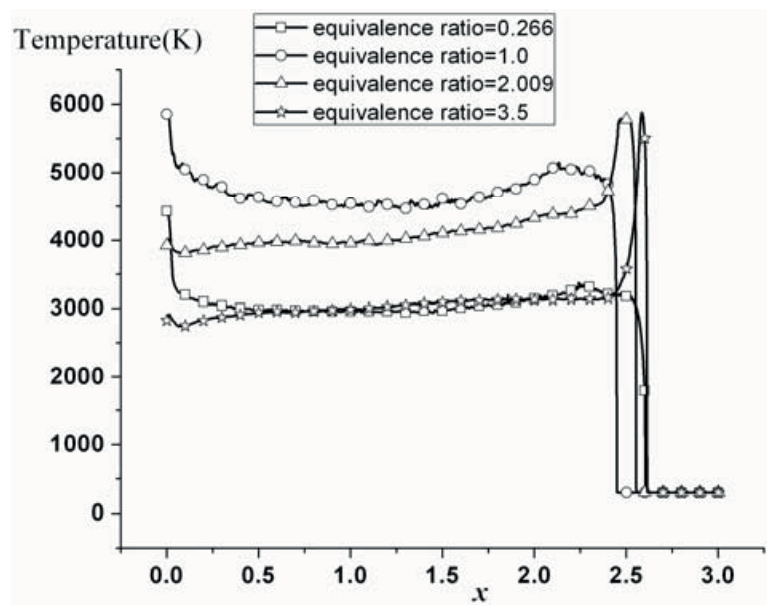

Figure 9. Temperature profiles of gas mixtures with different equivalence ratios. 


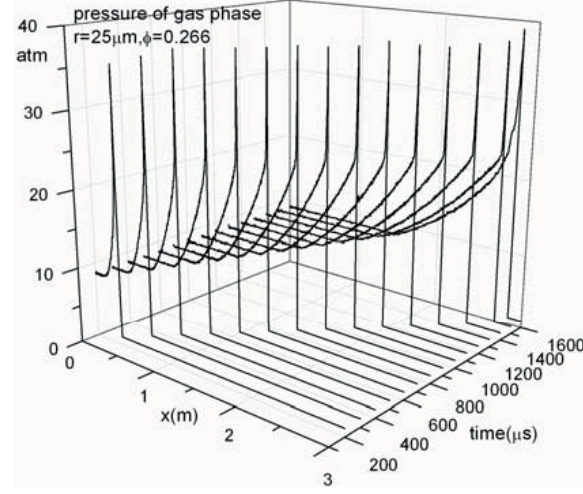

(a) pressure of gas phase $(\mathrm{r}=25 \mu \mathrm{m}, \Phi=0.266)$

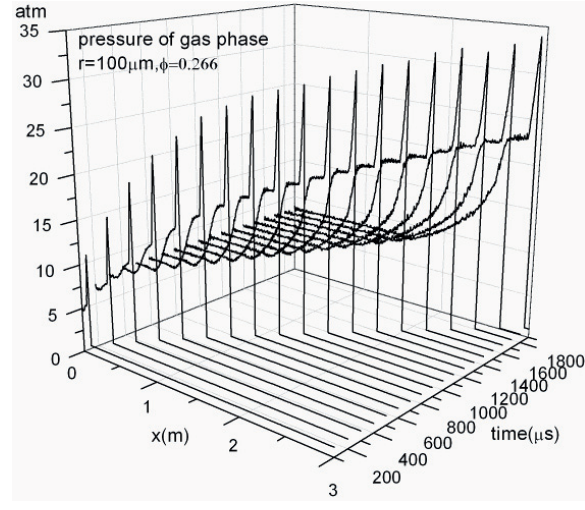

(c) pressure of gas phase $(\mathrm{r}=100 \mu \mathrm{m}, \Phi=0.266)$

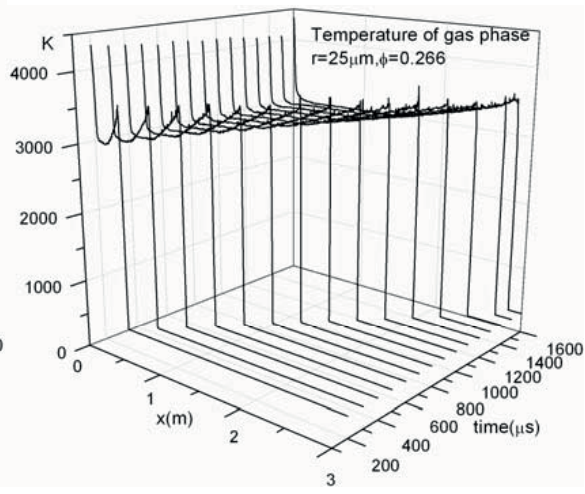

(b) temperature of gas phase $(\mathrm{r}=25 \mu \mathrm{m}, \Phi=0.266)$

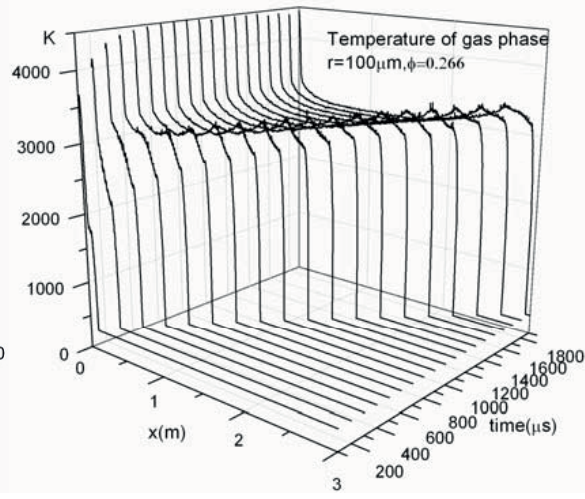

(d) temperature of gas phase $(\mathrm{r}=100 \mu \mathrm{m}, \Phi=0.266)$

Figure 10. Comparison of pressure and temperature profiles calculated with two different particle sizes $(\Phi=0.266)$.

onation front and the temperature of final products decrease in rich fuel mixtures.

\subsection{Influence of Particle Radius}

In this section, the influence of particle radius on two-phase detonations is discussed. Fuel-oxygen mixtures of three different equivalence ratios with different initial particle radii range from $5 \mu \mathrm{m}$ to $200 \mu \mathrm{m}$ are considered. Figure 10(a) (d) shows the comparison of pressure and temperature profiles calculated with two different particle sizes and the same equivalence ratio $\Phi=0.266$. In the mixture with finer particle size, the less time is spent to formatting steady self-sustained detonation wave. The peak temperature is behind the detonation front in mixtures with larger particle size. But the peak temperature appears at the detonation front in mixtures with finer particle size. That is because the finer particles are completely atomized between the shock and $\mathrm{C}-\mathrm{J}$ plane and react with oxygen, while the larger particles are not. Define the distance between the shock and C-J plane and the mass fraction of particle atomized between the shock and $\mathrm{C}-\mathrm{J}$ plane as efficient reaction length and efficient atomization ratio, respectively. In our simulations, we find that the efficient reaction length and the efficient atomization ratio are almost independent of equivalence ra- tios. Figure 11(a)-(c) shows the influence of particle radius on detonation velocity, efficient reaction length and the efficient atomization ratio. It can be seen that the reaction length almost increases linearly with particle radius. The detonation velocity is inversely proportional to the length of the reaction zone behind the shock front when the initial particle radius is greater than $50 \mu \mathrm{m}$. When the initial particle radius is less than $50 \mu \mathrm{m}$, the detonation velocity approaches a constant value and the efficient atomization ratio approaches to 1.0. It means that the chemical reactions occur between the shock and $\mathrm{C}-\mathrm{J}$ plane has significant influence on detonation propagation.

\section{Conclusion}

In this paper, an Eulerian-Lagrangian two-phase flow model is developed for liquid-fueled detonations. And an improved CE/SE scheme is applied to the numerical simulations. Detonations in liquid $\mathrm{C}_{10} \mathrm{H}_{22}-\mathrm{O}_{2}$ /air systems are simulated. It is proved that the present model keeps mesh convergence. It is also found that the propagation velocity in rich fuel mixtures is almost independent of the equivalence ratio, which is even higher than the theoretical C-J value of an all-gaseous mixture with very rich fuel. It is 


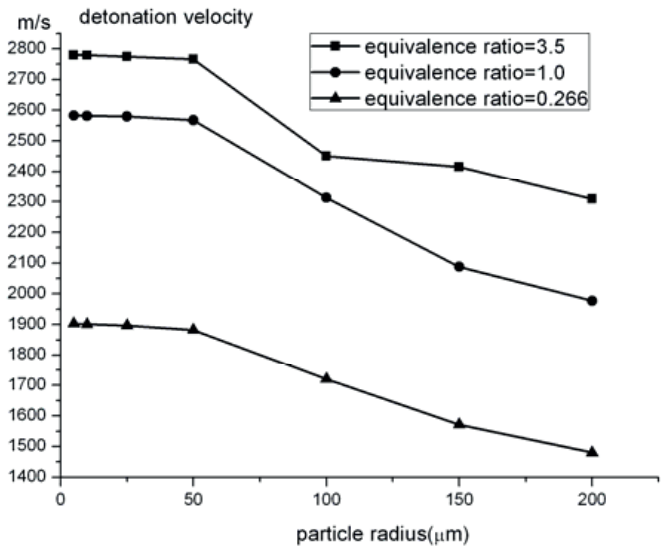

(a) detonation velocity versus particle radius

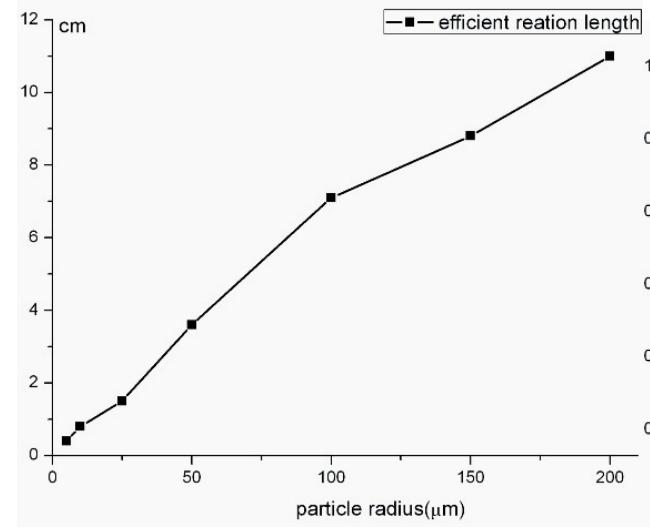

(b) efficient reaction length versus particle radius

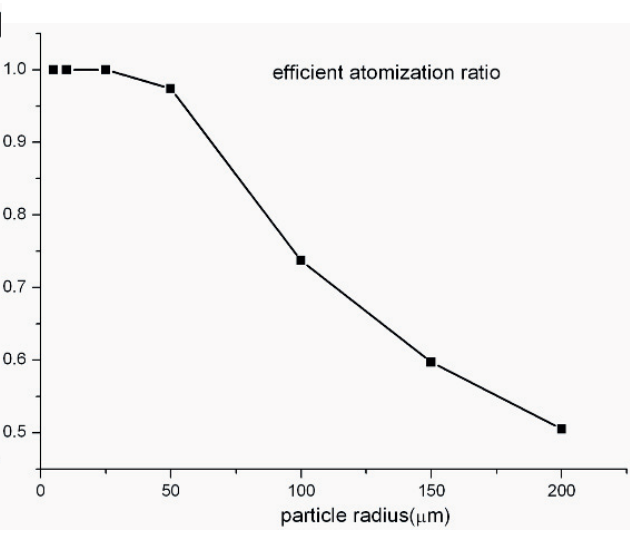

(c) efficient atomization ratio versus particle radius

Figure 11. The influence of particle radius on detonation parameters.

due to the difference between the global equivalence ratio and the efficient equivalence ratio of the gas-phase mixtures which sustains the detonation propagation. The influence of particle radius on liquid-fuel detonation is predicted by the present model. Detailed numerical results show that the detonation propagation velocity is mainly influenced by efficient reaction length and chemical reactions occur between the shock and C-J plane. In future work, the Mach reflections and cellular structures of liquid-fueled detonations will be further researched by the present model.

\section{Acknowledgments}

The authors gratefully acknowledge the financial support of the National Natural Science Foundation of China (Grant Nos. 10732010 and 10972010).

\section{References}

[1] E. K. Dabora, L. P. Weinberger, Present Status of Detonations in Two-Phase Systems, Acta. Astronaut. 1 (3-4) (1974) 361-372.
[2] A. Burcat, S. Eidelman, Evolution of a Detonation Wave in a Cloud of Fuel Droplets II. Influence of Fuel Droplets, AIAA J. 18 (11) (1980) 1233-1236.

[3] S. Eidelman, A. Burcat, The Mechanism of a Detonation Wave Enhancement in a Two-Phase Combustible Medium, P. Combust. Inst. 18 (1981) 1661-1670.

[4] S. A. Zhdan, Calculation of the Initiation of a Heterogeneous Detonation with a Charge of Condensed Explosive, Combust. Explo. Shock+ 17 (7) (1981) 674-679.

[5] D. V. Voronin, S. A. Zhdan, Calculation of Heterogeneous Detonation Initiation for a Hydrogen-Oxygen Mixture in an Explosion Tube, Combust. Explo. Shock+ 20 (5) (1985) 461-465.

[6] A. A. Vasil'ev, S. A. Zhdan, V. V. Mitrofanov, Detonation Initiation in Gaseous and Heterogeneous Systems, Gaseous and Heterogeneous Detonations: Science to Applications, edited by G. Roy, S. Frolov, K. Kailasanath, N. Smirnov, ENAS Publishers, Moscow, (1999) 25-38.

[7] S. A. Zhdan, E. S. Prokhorov, Calculation of the Cellular Structure of Detonation of Sprays in an H2-O2 System, Combust. Explo. Shock+ 36 (7) (2000) 777-784.

[8] N. N. Smirnov, V. F. Nikitin, J. Khadem, Sh. AlyariShourekhdeli, Onset of Detonation in Polydispersed FuelAir Mixtures, P. Combust. Inst. 31 (2007) 2195-2204.

[9] G. D. Roy, S. M. Frolovb, A. A. Borisovb, D. W. Netzer, Pulse detonation propulsion: challenges, current status, and 
future perspective, Prog. Energ. Combust. 30 (2004) 545672.

[10] K. Kailasanath, Liquid-Fueled Detonations in Tubes, J. Propul. Power. 22 (7) (2006) 1261-1268.

[11] A. A. Ranger, J. A. Nicholls, Aerodynamic Shattering of Liquid Drops, AIAA J. 7 (2) (1969) 285-290.

[12] S. Eidelman, A. Burcat, Evolution of a Detonation Wave in a Cloud of Fuel Droplet: Part I. Influence of Igniting Explosion, AIAA J. 18 (10) (1980) 1103-1109.

[13] C. K. Westbrook, F. L. Dryer, Simplified Reaction Mechanisms for the Oxidation of Hydrocarbon, Combust. Sci. Technol. 27 (1981) 31-43.

[14] T. M. Kiehne, R. D. Matthews, et al, An Eight Step Kinetics Mechanism for High Temperature Propane Flames, Combust. Sci. Technol. 54 (1987) 1-23.

[15] B. J. McBride, M. J. Zehe, S. Gordon, NASA Glenn Coefficients for Calculating Thermodynamic Properties of Individual Species, NASA/TP 2002-211556, Glenn Research Center, Cleveland, (2002).

[16] S.C. Chang, The Method of Space-Time Conservation Element and Solution Element-A New Approach for Solving the Navier-Stokes and Euler Equations, J. Comput. Phys. 119 (2) (1995) 295-324.

[17] S. C. Chang, X. Y. Wang, C. Y. Chow, The Space-Time Conservation Element and Solution Element Method: A New High-Resolution and Genuinely Multidimensional Paradigm for Solving Conservation Laws, J. Comput. Phys. 156 (1) (1999) 89-136.
[18] G. Wang, D. L. Zhang, K. X. Liu, An Improved CE/SE Scheme and Its Application to Detonation Propagation, Chin. Phys. Lett. 24 (2007) 3563-3566.

[19] J. T. Wang, K. X. Liu, D. L. Zhang, A New CE/SE Scheme for Multi-Material Elastic-Plastic Flow and Its Application, Comput. Fluids 38 (2009) 544-551.

[20] G. Wang, D. L. Zhang, K. X. Liu, J. T. Wang, An Improved CE/SE Scheme for Numerical Simulation of Gaseous and Two-Phase Detonations, Comput. Fluids 39 (2010) 168-177.

[21] G. Wang, D. L. Zhang, K. X. Liu, Numerical Study on critical Wedge Angle of Cellular Detonation Reflections, Chin. Phys. Lett. 27 (2010) 024701.

[22] Q. Y. Chen, J. T. Wang, K. X. Liu, Improved CE/SE scheme with particle level set method for numerical simulation of spall fracture due to high-velocity impact, J. Comput. Phys. 29 (2010) 7503-7519.

[23] K. W. Ragland, E. K. Dabora, J. A. Nicholls, Observed Structure of Spay detonations, Phys. Fluids 11 (11) (1968) 2377-2388.

[24] S. A. Gubin, M. Sichel, Calculations of the Detonation Velocity of a Mixture of Liquid Fuel Droplets and a Gaseous Oxidizer, Combust. Sci. Technol. 17 (1977) 109-111.

[25] M. J. Tang, J. A. Nicholls, M. Sichel, Z. C. Lin, The Direct Initiation of Detonation in Decane-Air and Decane-Oxygen Sprays, Gas dynamics laboratories, report No. UM-0184041, October 1983.

[26] B. McBride, S. Gordon, Computer program for calculation of complex chemical equilibrium compositions, rocket performance, Incident and Reflected shocks, and ChapmanJouguet Detonation, NASAsp-273, 1971. 